\title{
Network-Load Aware Adaptive Channel Access Control for WLAN*
}

\author{
Xiaoyan $\mathrm{Wu}^{1}$, Qinghe Du${ }^{1,2}$, Pinyi Ren ${ }^{1}$ \\ ${ }^{1}$ School of Electronic and Information Engineering, Xi'an Jiaotong University, Xi'an, China \\ ${ }^{2}$ National Mobile Communications Research Laboratory of Southeast University, Nanjing, China \\ Email:xywu09@stu.xjtu.edu.cn, duqinghe@mail.xjtu.edu.cn,pyren@mail.xjtu.edu.cn
}

Received June 2013

\begin{abstract}
Wireless local area network (WLAN) brings us a low cost and high bandwidth experience and thus plays a critically important role in current/future networks to support high-rate transmissions. To better provide quality-of-service (QoS) for WLAN users, we in this paper propose an improved scheme called "A-EDCA" (adaptive EDCA), based on enhanced distributed channel access (EDCA) of IEEE 802.11e under the infrastructure mode. Our proposed scheme aims at efficiently adapting the transmission over WLAN to the time-varying network conditions and mitigating the competition ability unfairness between access point (AP) and non-AP stations (STAs). Specifically, all non-AP STAs adaptively modify the contention window based on the network condition. Moreover, AP skips the backoff phase by setting its backoff counter as zero when non-AP STA completes transmission successfully to relieve the unfairness. At last, simulation results demonstrate the effectiveness of the proposed approach.
\end{abstract}

Keywords: Quality of Service (QoS); IEEE 802.11e; Enhanced Distributed Channel Access (EDCA)

\section{Introduction}

Due to the characteristics of the high bandwidth, low cost and easy deployment, WLAN has surrounded us everywhere. WLAN has two kinds of modes, one with AP and the other without AP. The former mode is adopted in the most practical deployments. Hence, we consider the mode with AP (also called infrastructure mode). Traditional IEEE 802.11 protocol offers us two access policies, namely, DCF (distributed coordination function) and PCF (point coordination function) [1]. DCF is a policy based on competition and PCF is based on polling. Both DCF and PCF cannot provide QoS guarantees. To accommodate QoS, IEEE 802.11e is proposed including EDCA (enhanced distributed channel access) mode and HCCA (HCF controlled channel access) mode [2]. Most network devices are based on EDCA due to its easy realization and good expansibility.

Therefore, we only discuss EDCA policy in this paper. In EDCA, some important parameters including AC (access

\footnotetext{
*The research reported in this paper was supported by the National Natural Science Foundation of China under Grant No. 61102078, the National Science and Technology Major Project under Grant No. 2013ZX03002003-004, the Specialized Research Fund for the Doctoral Program of Higher Education under Grant No. 20110201120014, the Open Research Fund of National Mobile Communications Research Laboratory, Southeast University (No. 2011D10), and the Fundamental Research Funds for the Central University.
}

category), AIFS (arbitrary inter-frame space) and TXOPlimit (transmission opportunity limit) are adopted. Different services have different parameters for QoS support. However, there are still some problems when using EDCA. Specifically, stations may suffer from performance degradations and radio resources are not fully utilized due to the fixed parameter settings. When the number of stations increases, the probability of collisions increases leading to frequent retransmissions and a decrease of the overall throughput [3]. In addition, there exists unfairness of channel access competition ability between AP and non-AP stations (STAs) which is because that AP almost has the same channel access competition ability with one non-AP STA while the number of non-AP STAs is far larger than that of AP. This causes that AP cannot handle the data in time.

For providing better QoS, many analytical approaches are proposed. Bianchi [4] developed a simple DTMC (discrete time Markov chain) and the saturation throughput was obtained by applying regenerative analysis to a generic slot time. In [5], authors represented an analytical model to analyze the performance of EDCA. Cali et al. [6] employed renewal theory to analyze a p-persistent variant of DCF with persistence factor $\mathrm{p}$ derived from the C.W. Tay et al. [7] instead used an average value mathematical method to model the DCF backoff procedure 
and to calculate the average number of interruptions that the backoff timer experienced. The throughput and delay for EDCA were derived based on Markov Chain [8,9]. In order to realize QoS guarantees, researchers have proposed many approaches. A large proportion of these approaches are based on EDCA [10-15].

In this paper, we propose an improved scheme "AEDCA" based on EDCA with a low computation complexity. Firstly, we adjust the contention window (CW) adaptively according to the current network load condition so as to make full use of the wireless channel resource. Secondly, we adjust channel access policy of AP.

The remainder of this paper is organized as follows: in Section 2, we present the system model and the brief summary of EDCA policy. The design and detailed procedures of our proposed scheme are given in Section 3. The performance analysis is given in Section 4. We show and analyze the simulation results in Section 5. Finally, we conclude our work in Section 6.

\section{System Model}

\subsection{System Model}

We consider the infrastructure mode of WLAN. There are $\mathrm{N}$ non-AP STAs distributed randomly and one AP in WLAN. Each non-AP STA transmits/receives packets through AP and there are no packet transmissions between non-AP STAs. We assume that the channel is ideal and that all STAs (including AP and non-AP STAs) are still for simplicity. The scenario is depicted in Figure 1. Without loss of generality, we consider two kinds of traffics: voice traffic and background traffic. As we know, voice traffic is delay-sensitive and has higher priority than background traffic. Because some problems exist when EDCA policy is used directly, we propose an improved channel access scheme called "A-EDCA".

\subsection{Enhanced Distributed Channel Access}

This subsection brie fly summarizes the operations ofIEEE

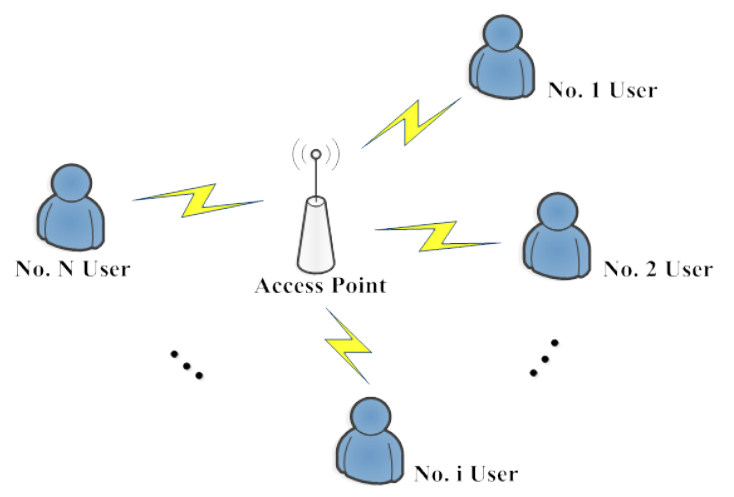

Figure 1. Scenario with N non-AP STAs and one AP. 802.11e EDCA. For detailed description, readers may refer to [1,2]. EDCA is an enhanced policy of DCF. There are some important notions, as depicted by Table 1. The packets from upper layer are classified into four ACs. Each AC is corresponding to one queue. Each STA has four queues and each queue has a backoff counter. The mapping from upper layer to medium access control (MAC) layer is shown as Figure 2 (refer to [2]). In EDCA, the channel access process is described as following. STA which has packets to send senses the channel. If the channel is idle for AIFS period, the STA transmits packets immediately. If the channel is busy, the backoff counter is initialized and set as a value based on the backoff mechanism. The STA enters the backoff phase after it senses the channel idle for AIFS period. In the backoff phase, the backoff counter decreases by one if the channel is idle for one time slot (TS) consecutively. If the channel becomes busy, the backoff counter is suspended. The counter resumes when the STA senses the channel idle for AIFS period again. The STA transmits packets if the backoff counter decreases to zero. The node can transmit multiple packets during TXOP. When the STA successfully transmits data, its $C W$ is set as $C W_{\text {min }}$ and its backoff counter is set as a value from $[0, C W-1]$ randomly. The channel access process is illustrated as Figure 3 (refer to [2]).

There exist two kinds of collisions in EDCA, namely, virtual collisions and inter-STA collisions. The former occurs when more than two queues (including two queues) in a STA simultaneously attempt to transmit packets.

Table 1. Notions and Comments.

\begin{tabular}{cc}
\hline Notion & Comment \\
\hline TS & time slot \\
SIFS & short inter-frame space \\
DIFS & distributed inter-frame space \\
AIFS & arbitrary inter-frame space \\
CW & contention window \\
CW $W_{\text {min }}$ & minimum contention window \\
AC & access category \\
TXOP & transmission opportunity \\
retry & maxim
\end{tabular}

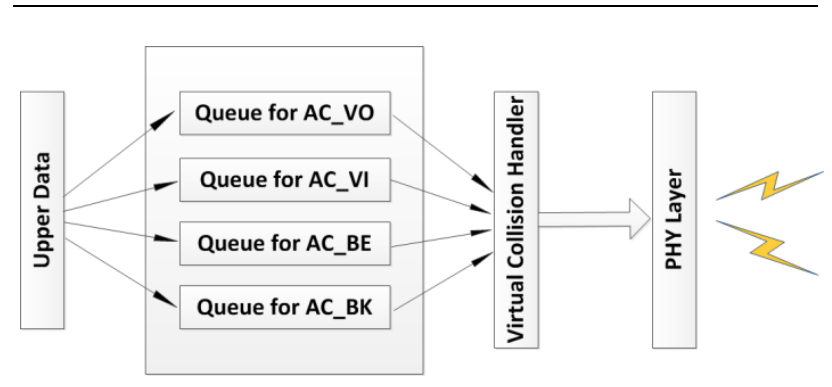

Figure 2. The mapping from upper layer to medium access control (MAC) layer. 


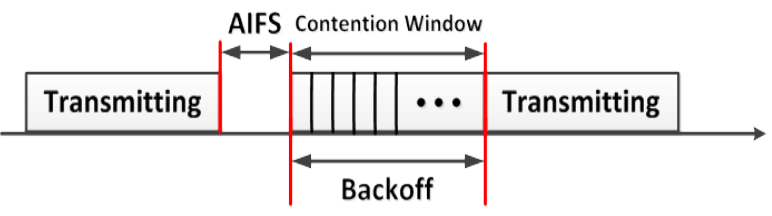

Figure 3. Channel Access Process in Enhanced Distributed Channel Access (EDCA).

The queue with the highest priority wins and attempts to transmit data while the others do not send data but update their CWs according to the backoff mechanism depicted in Equations (1)-(2) and continue to sense the channel.

$$
\begin{gathered}
C W_{\text {temp }}=2 *\left(C W_{\text {old }}+1\right)-1 \\
C W_{\text {new }}= \begin{cases}2 *\left(C W_{\text {old }}+1\right)-1, & C W_{\text {temp }} \leq C W_{\text {max }} \\
C W_{\text {max }}, & C W_{\text {temp }}>C W_{\text {max }}\end{cases}
\end{gathered}
$$

The latter collision happens when there are more than two STAs (including two STAs) simultaneously transmit data. The STAs participating in the collision all update their CWs according to the backoff mechanism depicted in Equations (1)-(2), and increase retry number (denoted by retry $_{\text {num }}$ ) by one. Then all stations sense the channel for the next attempt again.

\section{A-EDCA Channel Access Policy}

In this section, we present A-EDCA channel access policy. To begin with, we present the general idea of A-EDCA. Then the detailed description is given.

\subsection{Proposed A-EDCA Channel Access Policy}

There exist problems when EDCA policy is used directly. It cannot indicate how crowded the channel becomes that a certain station either succeeds to transmit packets or collides with others. In order to adapt to the network load condition, we have to find a metric that can reflect what the current load condition is like. From the backoff mechanism we know that the more crowded the WLAN is,

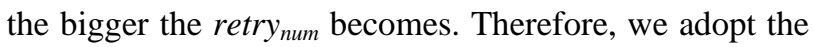
average value of retry num $_{\text {(denoted by } E[\text { retry }}$ num $]$ ) as the metric. In order to provide AP with the bigger channel access opportunity to relieve the unfairness, we adjust the channel access policy of AP. After a certain non-AP STA transmits packets successfully we set the backoff counter of AP as zero so as to make AP skip the backoff phase and attempt to transmit data in the next time.

\subsection{Detailed Description of A-EDCA}

\subsubsection{CW Value Adjustment}

When a certain STA (including AP) succeeds to transmit packets we calculate $E\left[\right.$ retry $\left._{\text {num }}\right]$ using Equation (3).

$$
\begin{aligned}
& E\left[\text { retry }_{\text {mom }}\right]=\frac{1}{M} \sum_{i=1}^{M} \text { retry }_{\text {mom }}(i) \\
& \text { s.t. } \operatorname{retry}_{\text {num }}(i)>0
\end{aligned}
$$

Here, $M$ is the number of stations whose retry $_{\text {num }}>0$. The channel is not busy if $E[$ retry num $]<\lambda$ and the channel becomes crowded if $E\left[\right.$ retry $\left.y_{\text {num }}\right] \geq \lambda$. We compare $E\left[\right.$ retry $\left.y_{\text {num }}\right]$ with the threshold (denoted by $\lambda$ ) and update CW according to Equation (4).

$$
C W= \begin{cases}C W_{\text {min }}, & E\left[\text { retry }_{\text {num }}\right]<\lambda, \\ C W, & E\left[\text { retry }_{\text {num }}\right] \geq \lambda .\end{cases}
$$

When collision occurs, we compute $E\left[\right.$ retry $\left._{\text {num }}\right]$ using Equation (3) and update the CW of the stations participating in the collision using Equations (1) (2) and (5).

$$
C W= \begin{cases}C W_{\text {new }}, & E\left[\text { retry }_{\text {num }}\right] \geq \lambda, \\ C W, & E\left[\text { retry }_{\text {num }}\right]<\lambda .\end{cases}
$$

\subsubsection{Channel Access Policy Adjustment for AP}

When a non-AP STA succeeds to transmit packets, AP sets its backoff counter as zero which makes AP skip the backoff phase. Then AP can attempt to transmit data after AIFS period during which the channel is continuously idle. This adjustment relieves the unfairness between the channel access competition ability of non-AP STAs and that of AP, depicted in Figure 4. We emphasize that the policy that we set backoff counter of AP as zero is adopted only when non-AP STA completes transmission successfully, which is for the sake of avoiding AP occupying the channel all the time.

The algorithm is presented in pseudo-code in Table 2.

\section{Performance Analysis}

The section is based on [8]. For simplicity, we only consider two ACs without loss of generality, namely, $\mathrm{AC}_{0}$ which presents background traffic and $\mathrm{AC}_{3}$ which presents voice traffic. We assume that each station only includes one AC. In order to obtain the expressions of performance metrics, some equations are given as follows. The probability $\tau_{i}$ that a station of $\mathrm{AC}_{i}$ transmits data in a slot is shown by

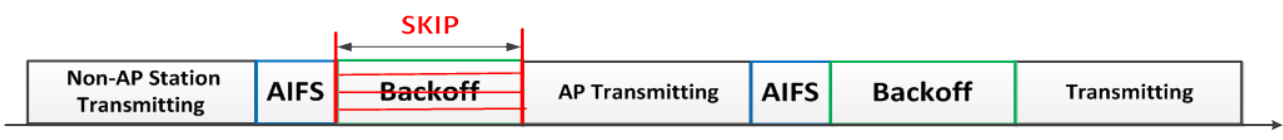

Figure 4. Channel Access Policy Adjustment of AP in A-EDCA. 
Table 2. A-EDCA Algorithm.

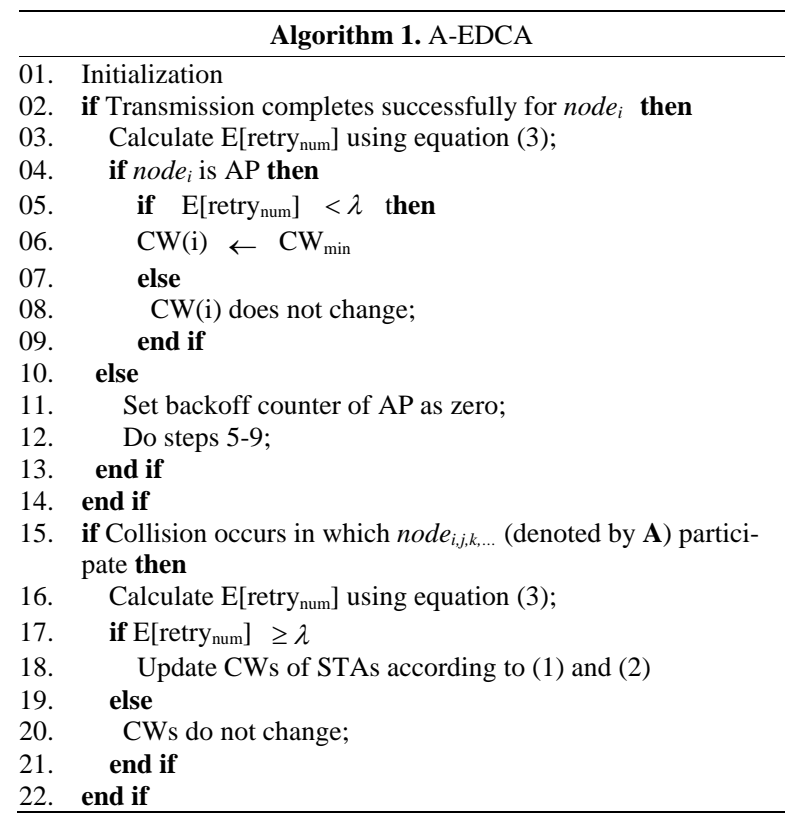

$$
\tau_{i}= \begin{cases}\frac{\left(1-p^{r+1}\right)}{(1-p) P_{I}} \beta_{0,0,0} & i=0, \\ \frac{1-p^{r+1}}{1-p} \alpha_{0,0} & i=3 .\end{cases}
$$

where $r$ is retry $y_{\text {lim }}, p$ is the collision probability that a frame encounters, $\beta_{0,0,0}$ and $\alpha_{0,0}$ are steady probabilities referred to [8], and $P_{I}$ is the probability that the channel is idle in a time slot, given by $P_{I}=q_{1} /\left(1+q_{1}-q_{2}\right)$. Here, $q_{1}$ and $q_{2}$ is the probability that there are no transmissions in a slot during an AIFS period and during other period respectively, depicted as follows:

$$
\begin{aligned}
& q_{1}=\left(1-\tau_{3}\right)^{n_{3}} \\
& q_{2}=\left(1-\tau_{0}\right)^{n_{0}}\left(1-\tau_{3}\right)^{n_{3}}
\end{aligned}
$$

where $n_{i}$ is the number of stations of $\mathrm{AC}_{i}$. The probability $P_{B}$ that a slot is busy is given by $P_{B}=1-P_{I}$. The probability $P_{s i}$ that a station of $\mathrm{AC}_{i}$ succeeds to transmit data in a slot time is shown by

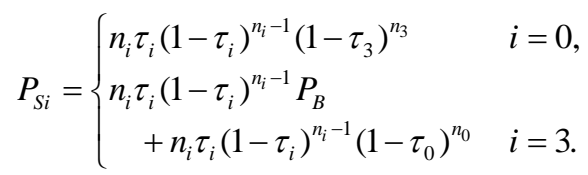

The probability $P_{C}$ that collisions happen in a slot time is given by $P_{C}=1-P_{I}-P_{S}$.

According to [8], throughput (denoted by $S$ ) is presented as follows:

$$
S=P_{S} * E[P] / E[T S]
$$

where $P_{S}$ is the probability that a successful transmission occurs in a slot time depicted by $P_{S}=P_{s 0}+P_{s 3}, E[P]$ is the average size of frame, and $E[T S]$ is the average length of a time slot given by $E[T S]=P_{I} \sigma+P_{S} T_{S}+$ $P_{C} T_{C}$. Here, $\sigma$ is the length of a time slot, $T_{S}$ is the average time of successful transmission, and $T_{C}$ is the average time of collision. The $T_{S}$ and $T_{C}$ in the basic mode are presented by

$$
\begin{aligned}
& T_{C}=H+E[P]+S I F S+A C K_{\text {timeout }} \\
& T_{S}=H+E[P]+S I F S+A C K+A I F S\left[\mathrm{AC}_{i}\right]
\end{aligned}
$$

where $H$ is the size including both MAC head and PHY head, $E[P]$ is the size of frame. From (9) we know that $S$ is a decreasing function of collision probability $p$.

The mean access delay (denoted by $E[D]$ ) is shown as follows:

$$
E[D]=\sum_{i=0}^{r}\left[\frac{\left(p^{i}-p^{r+1}\right) \frac{W_{i}+1}{2}}{1-p^{r+1}}\right] \cdot E[T S]
$$

We can derive that $E[D]$ is an increasing function of collision probability $p$.

By using A-EDCA policy without considering AP policy the collision probability can be decreased efficiently especially when the number of stations becomes large. From (9) (11) we can know that the throughput is improved and that delay is decreased by using A-EDCA policy. Taking AP policy adjustment into account, we can know that the downlink throughput can be improved and that the unfairness can be relieved.

\section{Simulation Results}

We adopt throughput, mean end-to-end delay and fairness of throughput to compare the performance between A-EDCA policy and EDCA policy. The fairness of throughput (denoted by I) is defined as the ratio of the difference between uplink throughput and downlink throughput to the throughput of uplink, depicted as Equation (12).

$$
I=\left(T_{\text {up }}-T_{\text {down }}\right) / T_{u p}
$$

where $T_{u p}$ and $T_{\text {down }}$ denote uplink throughput and downlink throughput, respectively.

We make some assumptions in our simulation. The channel is ideal and packet loss ratio (PLR) is only introduced by the event that the retransmission time is up

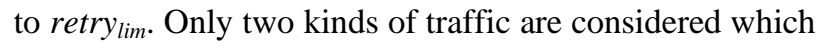
are voice traffic (denoted by $\mathrm{AC}_{3}$ ) and background traffic (denoted by $\mathrm{AC}_{0}$ ). TXOP is set as zero for all nodes, which means that the node occupying the channel successfully can only transmit one packet. We adopt the basic access mode. Simulation parameters are listed in Table 3.

Due to the dynamic adjustment of CW and AP channel access policy adjustment, the probability of collisions can 
be decreased and AP can get more opportunity to access the shared channel, which improves the performance of throughput, end-to-end delay and relieves the unfairness between AP and non-AP STAs.

Figure 5 shows the performance comparison between

Table 3. Simulation Parameters.

\begin{tabular}{|c|c|}
\hline Parameters & value \\
\hline PHY Header & 192 bits \\
\hline MAC Header & 272 bits \\
\hline SIFS & 1 Time Slot \\
\hline DIFS & 3 Time Slot \\
\hline $\operatorname{AIFSN}\left[\mathrm{AC}_{0}\right]$ & 7 \\
\hline AIFSN[AC $\left.\mathrm{AC}_{3}\right]$ & 2 \\
\hline ACK Frame & PHY Header +112 bits \\
\hline $\mathrm{ACK}_{\text {timeout }}$ & ACK Frame + DIFS \\
\hline Data Rate & $11 \mathrm{Mbps}$ \\
\hline Time Slot & $20 \mu \mathrm{s}$ \\
\hline retry $_{\text {lim }}$ & 7 \\
\hline $\mathrm{AC}_{0}$ & 1500 bytes \\
\hline $\mathrm{AC}_{3}$ & 200 bytes \\
\hline $\mathrm{CW}\left[\mathrm{AC}_{0}\right]$ & $\{31,63,127,255,511,1023\}$ \\
\hline $\mathrm{CW}\left[\mathrm{AC}_{3}\right]$ & $\{7,15,31,63,127,255\}$ \\
\hline
\end{tabular}

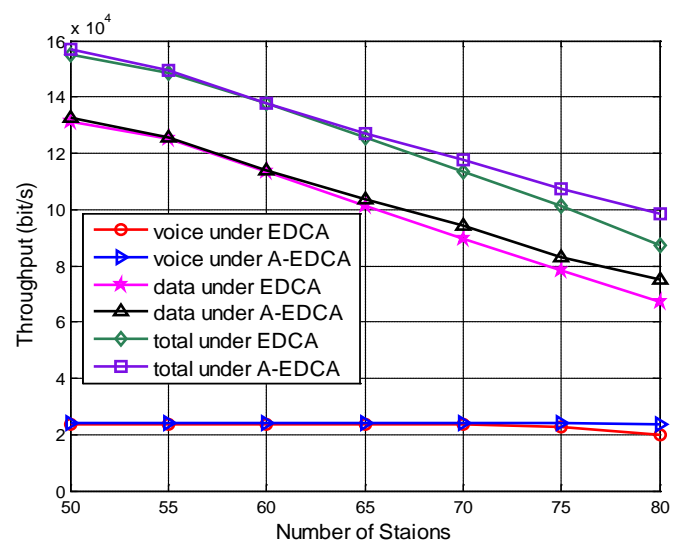

(a)

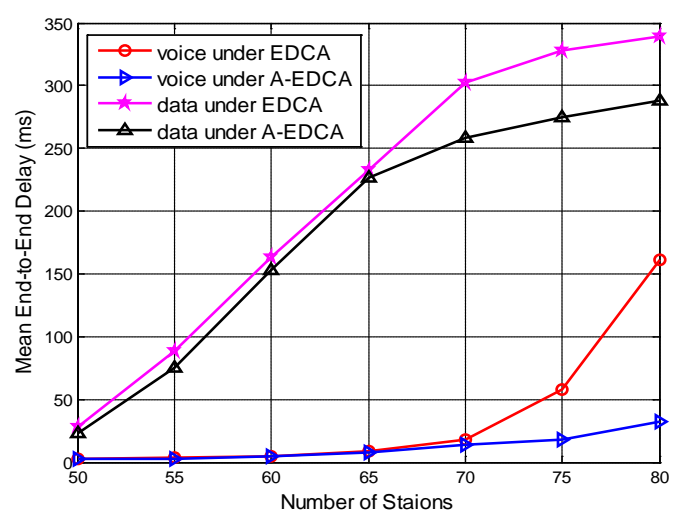

(b)

Figure 5. (a) Comparison of Throughput When No. of STAs Changes; (b) Comparison of Mean End-to-End Delay When No. of STAs Changes.
A-EDCA and EDCA when the number of stations changes. Figure 5(a) illustrates that throughput under A-EDCA is larger than that under EDCA. Figure 5(b) shows that mean end-to-end delay becomes smaller under A-EDCA. From Figures 6(a) and (b) we can see that our A-EDCA brings performance improvements when the packet arrival rate changes. The cumulative distribution function of mean end-to-end delay is shown in Figure 7(a). We can see that about 90 percent of delay is under $400 \mathrm{~ms}$ under A-EDCA policy while it is $500 \mathrm{~ms}$ under EDCA policy. Figure 7(b) shows us that the unfairness is relieved under A-EDCA especially when the number of stations is large.

\section{Conclusion}

This paper has considered the problem of IEEE 802.11e EDCA policy which includes not adapting to the timevarying network condition due to the fixed parameter settings and the channel competition ability unfairness between AP and non-AP STAs. In order to solve the problem, we proposed an improved scheme "A-EDCA".

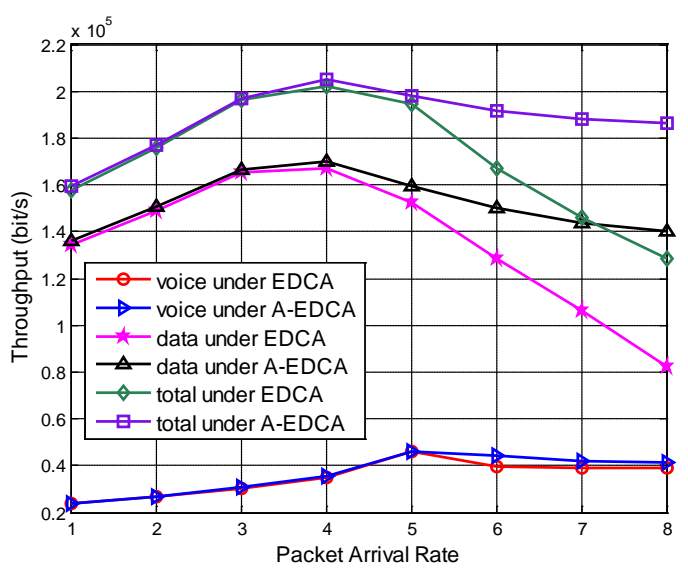

(a)

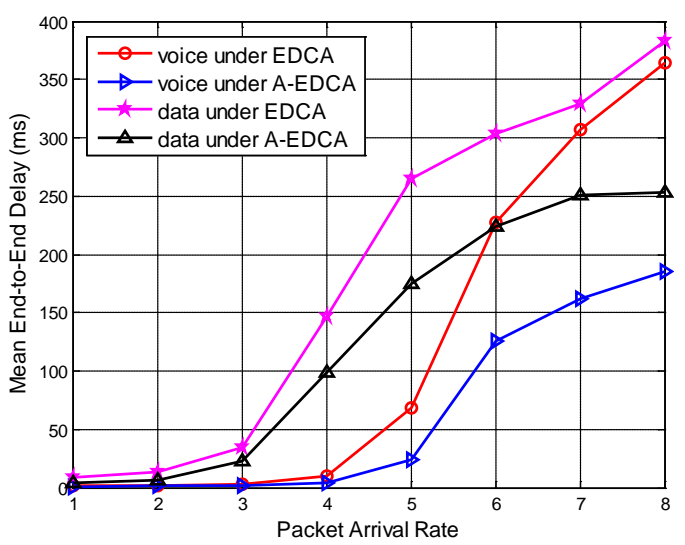

(b)

Figure 6. (a) Comparison of Throughput When Packet Arrival Rate Changes; (b) Comparison of Mean End-to-End Delay When Packet Arrival Rate Changes. 


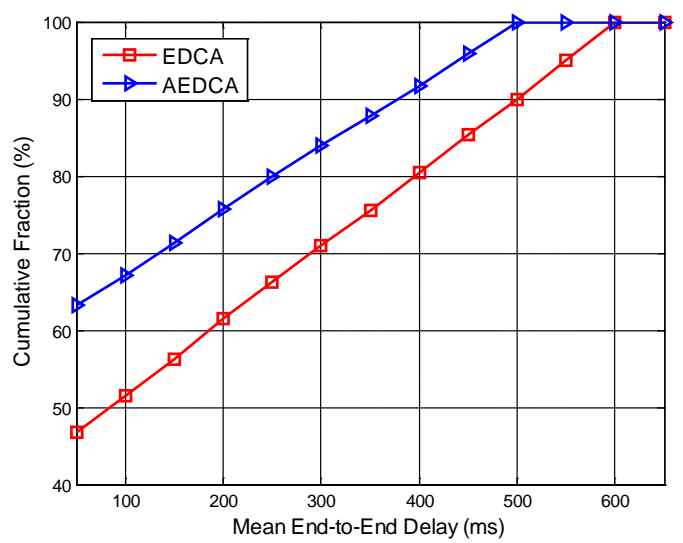

(a)

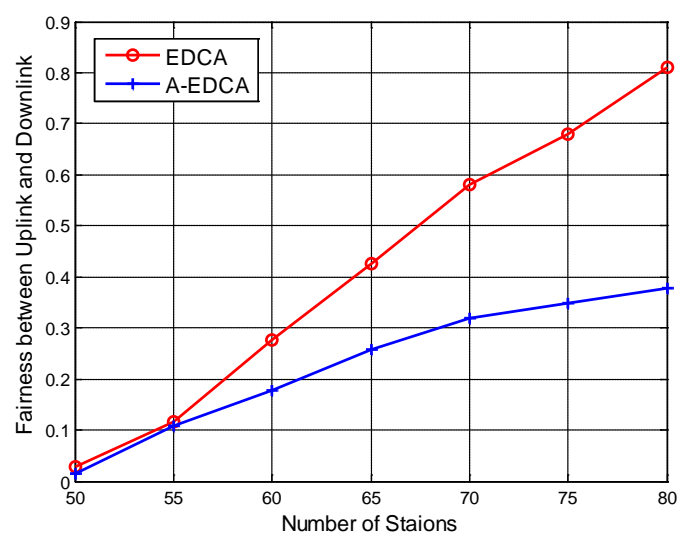

(b)

Figure 7. (a) Comparison for Delay CDF; (b) Comparison for Fairness.

In the scheme CW of the node is adjusted dynamically for the sake of adapting to the current network condition, and AP skips the backoff phase by setting its backoff counter as zero when non-AP STA completes transmission successfully to relieve the unfairness between channel access competition ability of AP and that of non-AP STAs. Through the simulations, we verify the effectiveness of our approach. The performances of both throughput and mean end-to-end delay are improved effectively. The unfairness is relieved to a large extent. Future research will focus on how to satisfy hard QoS requirements and how different kinds of access networks such as WLAN, LTE-A provide better services for users together.

\section{REFERENCES}

[1] Wireless LAN Medium Access Control (MAC) and Physical Layer (PHY) Specifications, ISO/IEC IEEE 802.11 Standard, 2005.

[2] Wireless LAN Medium Access Control (MAC) and Physical Layer (PHY) Specifications: Medium Access
Control (MAC) Quality of Service Enhancements, ISO/ IEC IEEE 802.11 Standard, 2005.

[3] F. Cali, M. Conti and E. Gregori, "IEEE 802.11 Wireless LAN: Capacity Analysis and Protocol Enhancement," 17th Annual Joint Conference of the IEEE Computer and Communications Societies, March 1998, pp. 142-149.

[4] G. Bianchi, "Performance Analysis of the IEEE 802.11 Distributed Coordination Function," IEEE Journal on Selected Areas in Communications, Vol. 18, 2000, pp. 535-547. http://dx.doi.org/10.1109/49.840210

[5] Z.-n. Kong, D. H. K. Tsang and B. Bensaou, "Performance Analysis of IEEE 802.11e Contention-Based Channel Access," IEEE Journal on Selected Areas in Communications, Vol. 22, No. 10, 2004, pp. 2095-2106.

[6] F. Cali, M. Conti and E. Gregori, "Dynamic Tuning of the IEEE 802.11 Protocol to Achieve a Theoretical Throughput Limit," IEEE Transactions on Networking, December 2000, pp. 785-799.

[7] J. C. Tay and K. C. Chua, "A Capacity Analysis for the IEEE 802.11 MAC Protocol,” Wireless Networks, Vol. 3, No. 3, 2001, pp. 159-171. http://dx.doi.org/10.1023/A:1016637622896

[8] J. W. Tantra, H. F. Chuan and A. B. Mnaouer, “Throughput and Delay Analysis of the IEEE 802.11e ED CA Saturation," IEEE International Conference on Communications (ICC), Vol. 5, May 2005, pp. 3450-3454,

[9] X. Yang, "Enhanced DCF of IEEE 802.11e to Support QoS,” IEEE WCNC, 2003.

[10] A. Veres, A. T. Campbell, M. Barry and L. H. Sun, "Supporting Differentiation in Wireless Packet Networks Using Distributed Control," IEEE Journal on Selected Areas in Communications, Vol. 19, No. 10, 2001, pp. 2081-2093.

[11] A. Leonovich and H.-W. Ferng, “A Time Slots Coordination Mechanism for IEEE 802.11 WLANs,” IEEE Communications Letters, Vol. 14, No. 4, 2010, pp. 360-362. http://dx.doi.org/10.1109/LCOMM.2010.04.092443

[12] G. W. Wong and R. W. Donaldson, "Improving the QoS Performance of EDCF in IEEE 802.11e Wireless LANs," IEEE Pacific Rim Conference on Communications, Computers and Signal Processing (PACRIM), Vol. 1, August 2003, pp. 392-396,

[13] P. Patras, A. Banchs and P. Serrano, “A Control Theoretic Approach for Throughput Optimization in IEEE 802.11e EDCA WLANs," Mobile Networks and Applications, Vol. 14, No. 6, 2009, pp. 697-708. http://dx.doi.org/10.1007/s11036-008-0121-X

[14] Y. Q. Dong, Y. Wang and Q. Xia, “A Load Adaptive IEEE 802.11e EDCA Backoff Scheme with Enhanced Service Differentiation,” IEEE International Conference on Communication Technology, November 2010, pp. 1056-1059,

[15] T. Nilsson and J. Farooq, "A Novel MAC Scheme for Solving the QoS Parameter Adjustment Problem in IEEE 802.11e EDCA," IEEE International Symposium on a World of Wireless, Mobile and Multimedia Networks, 2008, pp. 1-9. 